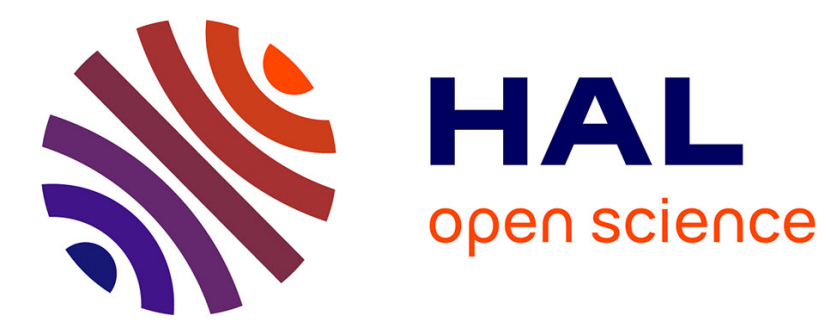

\title{
Le laboratoire national GANIL
}

\author{
M.G. Saint-Laurent
}

\section{To cite this version:}

M.G. Saint-Laurent. Le laboratoire national GANIL. Revue Generale Nucleaire, 1995, 5, pp.360-365. 10.1051/rgn/19955360 . in2p3-01524554

HAL Id: in2p3-01524554

https://hal.in2p3.fr/in2p3-01524554

Submitted on 18 May 2017

HAL is a multi-disciplinary open access archive for the deposit and dissemination of scientific research documents, whether they are published or not. The documents may come from teaching and research institutions in France or abroad, or from public or private research centers.
L'archive ouverte pluridisciplinaire HAL, est destinée au dépôt et à la diffusion de documents scientifiques de niveau recherche, publiés ou non, émanant des établissements d'enseignement et de recherche français ou étrangers, des laboratoires publics ou privés. 

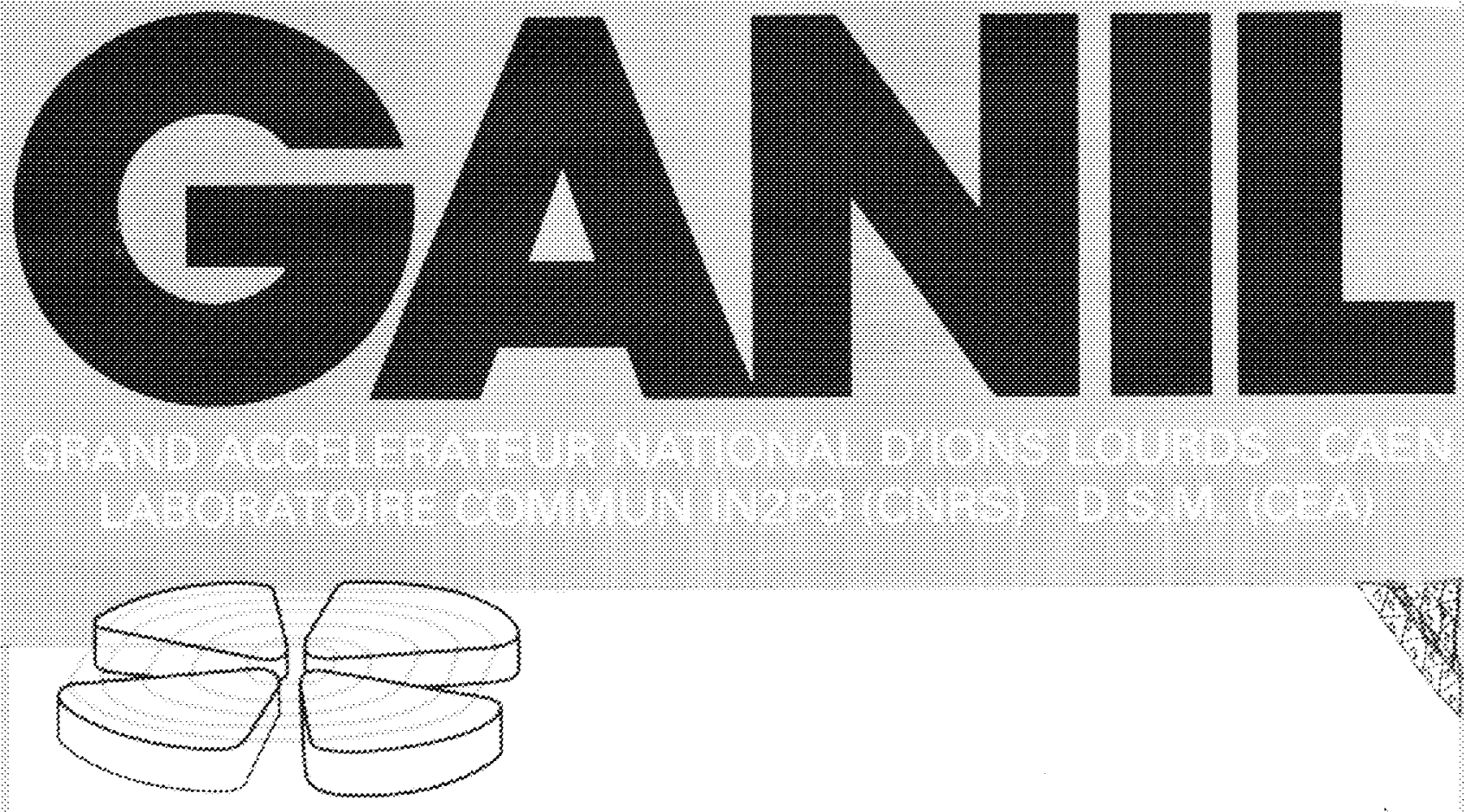

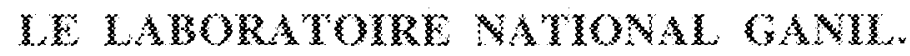

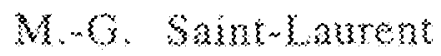

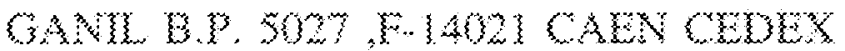

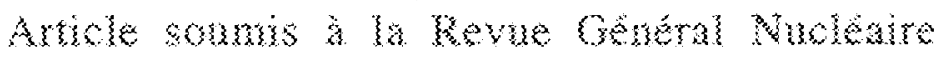

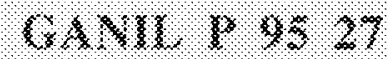




\section{LE LABORATOIRE NATIONAL GANIL. \\ M.-G. Saint-Laurent}

GANIL B.P. 5027 ,F-14021 CAEN CEDEX

Article soumis à la Revue Général Nucléaire

La science progresse souvent plus de l'anomalie observée que de la banalité. Créer des noyaux très perturbés en température, en proportion neutron - proton, en dimension...., observer ces espèces très éphémères, telles sont les prouesses réalisées au GANIL. La complexité observée de ces noyaux remet en question les interprétations théoriques les plus modernes.

\section{GANIL : LABORATOIRE D'ACCUEIL}

Laboratoire d'accueil mixte C.E.A. C.N.R.S., Le GANIL (Grand A ccélérateur $\mathbf{N}$ ational d'Ions $\mathbf{L}$ ourds) se veut un outil à la disposition de la communauté scientifique nationale et internationale. Depuis sa réalisation de 1975 à 1982, les expériences $s^{\prime}$ y succèdent aussi bien en physique nucléaire qu'en physique atomique, physique de la matière condensée ou en applications industrielles. Les physiciens, accueillis sur le site par une quinzaine de physiciens permanents, s'organisent en équipes souvent internationales : 700 visiteurs par an dont plus du quart viennent d'une dizaine de pays étrangers. Le label de grande installation scientifique européenne lui a été décerné.

\section{ACCÉLÉRER DES IONS LOURDS : LE GANIL.}

L'atome se compose d'un noyau au centre et d'électrons évoluant autour de ce coeur; Le noyau est constitué de nucléons : les neutrons et les protons. L'atome est électriquement neutre. Il est cependant possible de lui arracher des électrons et d'obtenir des atomes possédant une charge électrique non nulle, les ions.

Le GANIL est conçu pour accélérer un large éventail d'ions de l' Hélium à l' Uranium, Il leur communique une vitesse très élevée pouvant atteindre le tiers de la vitesse de la lumière. $L^{\prime}$ énergie maximale est de $95 \mathrm{MeV}$ par nucléon pour les ions légers (jusqu'à la masse 40) et de $25 \mathrm{MeV}$ par nucléon pour les plus lourds (Uranium). L' intensité de ces faisceaux est pour les ions les plus légers de l'ordre de $10^{12}$ particules par seconde et pour les ions les plus lourds de l'ordre de $10^{9}$ particules par seconde. 
Les principales étapes ( Fig. 1. ) de la production d'un faisceau GANIL sont :

- La production d'ions. Une source génère des ions faiblement chargés. Une différence de potentiel entre la source et le reste de l'installation permet l'extraction de ces ions. Ils sont ensuite accélérés dans un petit cyclotron $\mathrm{C} 01$ ou $\mathrm{C} 02$.

- La première accélération. Dans le cyclotron CSS1, les ions subissent une succession d'accélérations en traversant de nombreuses fois le même champ électrique. Un électroaimant crée un champ magnétique pour guider les ions sur des trajectoires circulaires.

- Le changement de la charge de l'ion. Après extraction du cyclotron CSS1, les ions traversent à grande vitesse une mince feuille de Carbone. Des électrons sont arrachés et les ions acquièrent une charge beaucoup plus grande que leur charge initiale.

- La seconde accélération. Grâce à cet accroissement de charge, un deuxième cyclotron CSS2 identique au premier peut à nouveau accélérer les ions et cela de façon beaucoup plus efficace.

- L'analyse magnétique. Le faisceau, soigneusement aligné, est finalement analysé en énergie par le spectromètre à haute résolution en "alpha", puis envoyé dans l' une des 8 salles d'expériences.

\section{L' ART DE CASSER DES NOYAUX POUR MIEUX LES COMPRENDRE :}

L'objectif du GANIL est d'étudier des noyaux atomiques fabriqués dans des conditions extrêmes, afin d'explorer la nature fondamentale de la matière nucléaire.

L'observation directe du noyau est impossible en raison de sa faible dimension $\left(10^{-13} \mathrm{~cm}\right)$. Il faut donc soit :

- agir sur lui et déduire sa structure des conséquences de la perturbation engendrée (physique des noyaux dits chauds).

- soit, s'il est radioactif, comprendre sa structure à partir de l' observation de son mode de radioactivité (physique des noyaux dits exotiques).

Des noyaux projectiles lancés à très grande vitesse ( le tiers de la vitesse de la lumière) interagissent avec les noyaux constituant une feuille de matière appelée cible. Des détecteurs placés autour de la cible mesruret les produits issus du choc. Ces collisions peuvent être classées selon leur "paramètre d'impact", distance qui 
sépare les centres des noyaux projectile et cible au moment de la collision :

- Si le paramètre d'impact est très faible la collision sera frontale et donc très violente. Une grande partie de l'énergie cinétique du noyau projectile est dissipée sous forme d'énergie thermique donnant naissance à des noyaux chauds ; la température de ces noyaux peut atteindre 6-8 MeV (1 $\mathrm{MeV}$ équivaut à environ 10 Milliards de degré) ; Leurs vies sont infiniment courtes : de l'ordre de $10^{-22}$ seconde.

L' objectif fondamental de la physique des noyaux chauds est de déterminer l'équation d'état reliant les différents paramètres température, pression et densité - pour la matière nucléaire.

Les résultats les plus significatifs indiquent que:

* Les températures maximales (énergies d'excitation) que peut supporter le noyau, sans se désintégrer totalement, excèdent nettement les prédictions théoriques.

* Le temps de vie des noyaux chauds, de l'ordre de 10-22 Seconde, est mesurable indirectement.

* La compréhension des mécanismes de dépôt d'énergie dans le noyau, doit prendre en compte d' une part les collisions individuelles entre les nucléons qui forment le projectile et la cible, d' autre part le fait que ces nucléons forment un noyau donc agissent collectivement. Des fluctuations complexes autour des valeurs moyennes sont ainsi responsables d'effets particuliers.

* À l'extrême, le noyau peut ne plus exister et se fragmenter en de nombreux et très petits morceaux ; Cette "vaporisation" du noyau, observé pour la première fois au GANIL est un phénomène très rare : 3 événements sur plus de10 millions de collisions.

Pour remonter au scénario de la collision, le physicien doit mesurer la totalité des débris produits dans une collision, en identifiant leur nombre atomique, leur masse, leur énergie cinétique, collision par collision. Les détecteurs doivent couvrir la quasi-totalité de l'espace autour de la cible, avoir une résolution suffisante en énergie mais aussi en angle d'émission, ainsi que des seuils de détection très faibles afin de détecter au mieux tous les produits de la réaction. GANIL est doté d'un parc de détecteurs variés et complémentaires : ORION pour les neutrons, Nautilus $\left(1^{\circ}\right.$ génération) et maintenant INDRA $\left(2^{\circ}\right.$ génération) ( Fig. 2 ) pour les 
particules chargées, le détecteur Franco-Allemand TAPS pour les photons gammas de haute énergie, les pions et les kaons.

- Pour des paramètres d'impact intermédiaires, les collisions seront périphériques et conduiront principalement à la "fragmentation" d'un ou des partenaires de la réaction : Les noyaux projectile et cible se recouvrent partiellement. Une partie du projectile arrache les nucléons de la cible qui se trouve sur sa trajectoire et forme avec eux une zone fortement excitée de nucléons, qui se désintègre par émission de particules légères. Le fragment de la cible qui ne participe pas à la réaction reste pratiquement au repos. Le reste du projectile, qui n'a pas contribué à la collision, poursuit son chemin avec la vitesse et la direction initiales du projectile. Lors du contact des noyaux projectile et cible, l'interaction ne se résume pas à une simple cassure de ces noyaux. Des échanges de nucléons de la cible vers le projectile ont lieu. Des noyaux exotiques, $c$ ' est à dire ayant un fort déséquilibre entre le nombre de neutrons et de protons par comparaison aux noyaux stables, peuvent être formés parmi ces fragments du projectile ou de la cible. Ces noyaux exotiques sont radioactifs.

\section{LA PHYSIQUE DES NOYAUX EXOTIQUES.}

Les modèles théoriques décrivent les noyaux comme un ensemble de nucléons interagissant mutuellement ; Le noyau n'est pas figé, ses nucléons peuvent se déplacer individuellement ou au contraire avoir un mouvement collectif de rotation ou de vibration. La nature des forces entre nucléons est mal connue. Les différentes théories existantes sont basées sur les propriétés des noyaux stables ( c.a.d. existant dans la nature). Ces théories présentent de grandes divergences dans leurs prédictions respectives concernant les noyaux exotiques. La confrontation avec les résultats expérimentaux permet de trancher entre les différentes forces utilisées dans les modèles et d'affiner les théories.

Avant de pouvoir étudier la structure d'un noyau radioactif, le physicien doit le créer et le conduire vers son dispositif d'observation. Cette étape comporte plusieurs phases :

- La production des noyaux radioactifs par une réaction de fragmentation et le meilleur choix du couple cible-projectile.

- La collecte rendue encore plus efficace grâce au dispositif SISSI (Source d'Ions Secondaires à Solénoïdes Intense).

- Le filtrage parmi la multitude des autres noyaux plus stables produits grâce aux différents spectromètres magnétiques : 
* Alpha dont un des principaux avantages est de trier et de distribuer les ions radioactifs dans toutes les salles expérimentales.

* SPEG (S pectromètre à Perte d'É nergie G ANIL) dont la qualité principale est la très haute résolution en énergie.

* LISE3 (Ligne Ions Super Epluchés) dont l' atout majeur est de pouvoir guider des ions très excédentaires en neutrons.

- Le tri recherché peut être plus sévère. Deux autres critères de tri sont alors employés, l'un tirant parti du ralentissement des ions par traversée de matière, l'autre utilisant un filtre de Wien (champs électrique et magnétique croisés) sélectionnant en vitesse.

- L' identification de l'ion radioactif par son numéro atomique, sa masse et son taux de comptage à l'aide d'un détecteur.

Une fois ces étapes préliminaires franchies, le physicien peut commencer l'étude des caractéristiques du noyau d'intérêt :

- soit en l'arrêtant complètement dans de la matière pour des mesures de type radioactivité (période radioactive, schéma de désintégration, masse...)

- soit en le faisant interagir avec une seconde cible pour des mesures nécessitant une perturbation de ce noyau (diffusion élastique, section efficace de réaction, dissociation des noyaux à halos, excitation coulombienne...).

Nous exposons brièvement dans la suite trois exemples extraits des nombreux sous-thèmes étudiés au GANIL concernant les noyaux exotiques :

- l'existence des noyaux exotiques

- leur masse

- les noyaux à halos.

L'EXISTENCE D'UN NOYAU EXOTIOUE, LA RECHERCHE DE L ' ÉTAIN DE MASSE 100.

La connaissance, pour chaque isotope, du nombre de neutrons minimum et maximum ( limite de stabilité) qu' il peut contenir tout en maintenant sa cohésion est la première information que doit être capable de prévoir un modèle.

Plus de 85 nouveaux noyaux ont été produits pour la première fois au GANIL. Parmi eux :

- des noyaux aussi déficients en neutrons que le Silicium de masse 22 ( seulement 8 neutrons pour 14 protons) ou aussi riches en neutrons que le carbone de masse 22 (16 neutrons pour 6 protons).

- le Néon de masse 29 dont la mise en évidence de son existence a conduit les théoriciens à revoir leurs modèles : La non- 
existence du noyau Néon de masse 29 était prédite par tous les modèles théoriques et les formules semi-empiriques de masse des noyaux à l'exception de celle des physiciens japonais Uno et Yamada.

La limite d'existence d'un noyau est atteinte expérimentalement du côté riche en neutrons seulement de l'hydrogène à l'azote, alors que, du côté déficient en neutrons, elle est connue de l' hydrogène au cuivre. Cette différence s'explique par le fait que, pour un isotope donné, il y a beaucoup plus de noyaux excédentaires en neutrons donc plus "éloignés" de la stabilité que de noyaux déficitaires en neutrons. Il est donc plus facile de former, pour un élèment donné, le plus léger de ses isotopes que le plus lourd.

Les progrès réalisés dans les techniques mises en oeuvre pour produire, collecter et séparer les noyaux exotiques portent tous leurs fruits avec la mise en évidence ( aux laboratoires GSI en Allemagne et GANIL en France) du noyau Étain de masse 100 ( Fig. 3 ) qui était activement recherché depuis 25 ans. En effet, il est l' unique des seuls cinq noyaux "doublement magiques avec un nombre égal de neutrons et de protons" à être situé aux confins de la stabilité - Un noyau est magique s'il possède un nombre bien caractéristique de neutrons ou de protons pour lesquels les propriétés du noyau ( masse, abondance naturelle...) présentent des variations brusques par rapport aux propriétés des autres noyaux de masse voisine, un noyau "doublement magique" l'est en neutrons et en protons. En particulier, ces noyaux magiques sont beaucoup plus stables. - Cette propriété se traduit par une structure en couches du noyau analogue à celles des niveaux atomiques pour le cortège électronique des atomes. L'étude ultérieure des caractéristiques de l'Étain de masse 100 : masse, période, mode de désintégration devrait permettre de répondre aux questions suivantes :

- la notion de stabilité renforcée auprès des couches magiques est-elle toujours valable loin de la stabilité ?

- Le rôle des neutrons et des protons est-il symétrique ?

- Qu'elle est l'influence mutuelle des neutrons et des protons pour un noyau doublement magique aussi exotique?

L'étape suivante concerne la recherche de l'éventuelle existence d'un autre noyau doublement magique extrêmement riche en neutrons, l' Oxygène de masse 28, lui aussi aux confins de la stabilité mais comportant un nombre différent de néutrons et de protons (20 neutrons et 8 protons). 


\section{LA MASSE D' UN NOYAUEXOTIOUE:}

D'autres mesures systématiques concernent les mesures spectroscopiques : masse, période nucléaire, type de radioactivité...

La masse d'un noyau est une information très précieuse qui reflète directement sa structure nucléaire : toute variation brusque indique un changement de structure ou une évolution dans la forme des noyaux. Le noyau est un objet physique comportant trop de constituants pour qu'une description détaillée microscopique puisse être réalisée, mais il en comporte aussi trop peu pour qu'une description macroscopique soit pleinement adéquate. Les différentes théories sont basées sur des forces nucléaires ellesmêmes déterminées à partir des propriétés des noyaux stables. Les mesures systématiques de masse mettent sévèrement à l'épreuve les modèles théoriques. Elles cernent aussi les noyaux présentant des particularités.

Des mesures de masse très précises ont été obtenues grâce à l'idée ingénieuse d' associer les deux spectromètres à haute résolution Alpha et Speg : Entre ces deux spectromètres, les noyaux parcourent une centaine de mètres en quelques millionièmes de seconde. En mesurant le temps de parcours des noyaux, avec une précision de quelques dixièmes de milliardièmes de seconde, le physicien détermine la vitesse des noyaux. En associant cette mesure à celle très précise de leur énergie cinétique, il en déduit la masse atomique avec une précision relative proche du millionième. Deux résultats majeurs concernent :

- la confrontation très contraignante ( Fig. 4 ) de ces mesures obtenues pour plus de cinquante noyaux excédentaires en neutrons aux modèles théoriques et la remise en question de ces modèles. Des mesures de masse pour les noyaux déficitaires en neutrons sont en cours d'analyse ; elle devrait compléter ces tests des modèles théoriques.

- la confirmation que les noyaux au voisinage du magnésium de masse 32 (ayant 20 neutrons au lieu de 12 pour le magnésium stable) sont déformés et non sphériques comme le sont les noyaux stables possédant 20 neutrons : Un noyau exotique possédant 20 neutrons n'est plus magique comme l'est un noyau stable comportant 20 neutrons.

Cette perte de la "magicité" pour ce noyau exotique soulève la question de nouveaux nombres magiques loin de la stabilité.

La résolution obtenue avec l'ensemble des spectromètres Alpha plus SPEG atteint ses limites pour les noyaux plus lourds, au delà de la masse 100. Pour augmenter la précision, il faut faire "voler" les noyaux sur une distance encore plus grande. C'est ce qui a été réalisé en utilisant comme base de temps de vol, les 
nombreux tours ( soit une distance de $2-3 \mathrm{~km}$ ) qu'une particule fait dans le 2 ème cyclotron CSS2 du GANIL. Cette méthode consiste à produire des noyaux exotiques par réaction de fusion des noyaux projectile et cible, suivie de l' évaporation de quelques nucléons. Pour cela une cible est placée entre les 2 cyclotrons CSS du GANIL. L'énergie incidente disponible en sortie du premier cyclotron CSS 1 est plus favorable à ce type de réaction qu' à une fragmentation du projectile. Cette technique est maintenant opérationnelle avec une précision du millionième. Elle a nécessité de surmonter deux difficultés principales :

- faire fonctionner une sonde nucléaire dans un environnement aussi défavorable que $l^{\prime}$ intérieur d'un cyclotron (forts champs électrique et magnétique).

- connaître exactement la trajectoire des ions dans le cyclotron.

La mesure de la masse du noyau doublement magique Étain de masse 100 est le prochain défi technique du GANIL.

\section{LES NOYAUX À HALOS:}

Parmi les noyaux légers très saturés en neutrons, le physicien rencontre des phénomènes tels les noyaux à halos (Fig. 5) : les neutrons excédentaires s'aventurent loin du "coeur" habituel de nucléons, bien au delà des dimensions nucléaires usuelles. A titre d'exemple, un neutron du lithium de masse 11 peut s'éloigner à plus de 10 fermis ( 1 fermi $=10^{-15} \mathrm{~m}$.) du centre de ce noyau alors que le rayon d' un noyau de masse équivalente tel le Carbone stable de masse 12 est seulement de 2.5 fermis, et que celui d'un noyau beaucoup plus lourd comme le plomb comportant 208 nucléons est de l'ordre de 7 fermis. Du point de vue de la physique quantique, les neutrons du halo ont pendant un bref laps de temps une probabilité non nulle de se trouver hors de la portée de l'interaction forte, interaction régnant entre les nucléons du noyaux et qui les retient à l'intérieur du noyau.

Comment le physicien a-t-il accès à la mesure de la taille des noyaux ?

Plus un noyau est gros, plus il a de chances $d$ ' interagir avec un autre noyau. C'est par des mesures de cette probabilité de réaction (section efficace de réaction) que le physicien peut déterminer la dimension du noyau.

Le noyau à halo, en présence d'un noyau cible, a une très forte probabilité de dissocier les nucléons du halo de son coeur. La détection des nucléons à différents angles donne directement des renseignements sur le halo. 
Le physicien détermine la densité de matière par des expériences plus difficiles de diffusion élastique. Pour cela il joue au billard avec les noyaux : il projette un noyau radioactif sur un noyau cible et il observe à quels angles ceux des ions incidents qui n'ont pas été modifiés en nature et en énergie par le choc, sont diffusés ; la distribution des noyaux détectés à différents angles le renseignent sur la façon dont se répartit la densité des nucléons à l'intérieur du noyau.

Ainsi, les noyaux Béryllium de masse 11, Carbone de masse 19 (halo formé de 1 neutron), Lithium de masse 11 , Béryllium de masse 14 (halo formé de 2 neutrons) et Bore de masse 8 (candidat éventuel pour un halo à 1 proton) ont été étudiés au GANIL. Par des mesures de sections efficaces de dissociation du halo dans les champs coulombien et nucléaire, de diffusion élastique, il a largement contribué à la connaissance actuelle qu'on a des noyaux à halos légers riches en neutrons :

- Le halo est bien dû aux neutrons excédentaires.

- L' extension du halo dépend de l'énergie qui relie le(s) nucléon(s) du halo au coeur : plus l'énergie de liaison est faible plus les nucléons peuvent s'éloigner du coeur et plus le halo est étendu.

- Le noyau à halo de 2 neutrons soutend la même idée de base que le noyau à halo de 1 neutron, à la restriction près que la présence de deux nucléons supplémentaires hors du coeur nécessite la résolution compliquée d'un système quantique à 3 corps (problème théorique non encore résolu). Pour les noyaux à halos de 2 nucléons (par ex : $\mathrm{Li}$ de masse 11) aucun des 3 sous-systèmes binaires ( par ex : ${ }^{9} \mathrm{Li}$ +neutron1, ${ }^{9} \mathrm{Li}+$ neutron2, neutron 1+neutron2) qui le composent ne sont liés; Il faut l'ensemble des 3 participants pour que la stabilité soit assurée.

Du fait de sa dimension plus importante, le noyau à halo aurait une probabilité beaucoup plus importante à basse énergie de fusionner avec un autre noyau. Là aussi, le GANIL a démarré un programme de recherches car ce sera peut-être la voie de demain pour produire des éléments très lourds au delà de l'Uranium.

\section{UN PAS DANS L'ASTROPHYSIQUE NUCLÉAIRE :}

La physique des noyaux exotiques a également de très nombreuses implications dans notre compréhension du cosmos, car c'est via les noyaux exotiques que les éléments lourds qui composent notre univers se sont formés.

Les réactions nucléaires jouent un rôle capital dans l'évolution de l'univers. Elles sont à l'origine de la formation des éléments qui constituent l'univers à travers les différents processus de la 
nucléosynthèse stellaire. Les réactions de ce qui est appelé le cycle CNO chaud sont particulièrement critiques dans les calculs d'évolution stellaire explosive des novae et des supernovae. Ces réactions sont difficiles à étudier car elles font intervenir des noyaux radioactifs à de très basses énergies que l'on ne sait que très difficilement atteindre. Deux d'entre elles : $13 \mathrm{~N}+$ proton --> $14 \mathrm{O}+\gamma$ et ${ }^{11} \mathrm{C}+$ proton $-->12 \mathrm{~N}+\gamma$ ont pu l' être à GANIL grâce à un stratagème qui consiste à les étudier par le biais des réactions inverses, en dissociant les noyaux $14 \mathrm{O}$ et $12 \mathrm{~N}$ dans le champ coulombien d'un cible : $14 \mathrm{O}+\gamma \rightarrow 13 \mathrm{~N}+$ proton et $12 \mathrm{~N}+\gamma \rightarrow 1^{1} \mathrm{C}$ + proton. Ces réactions, pour être équivalentes, doivent être réalisées à des énergies élevées, énergies que l'on sait atteindre à GANIL.

Les grandes lignes du scénario de nucléosynthèse stellaire sont connues. Si l'on sait que différents cycles de combustion sont responsables de la nucléosynthèse des éléments jusqu'au Fer, le passage d'un cycle à l'autre est encore mal compris. Là encore, le GANIL a joué un rôle essentiel en déterminant les énergies possibles ("schémas de niveau") que peut prendre l'isotope de Sodium de masse 20 en étudiant la décroissance radioactive du Magnésium de masse 20. Ces données sont essentielles pour comprendre comment s'effectue le passage du cycle CNO (Carbone, Azote, Oxygène) au cycle suivant $\mathrm{Ne}-\mathrm{Na}$ (Néon, Sodium).

Lors de l'explosion de supernovae, un énorme flux de neutrons est émis. De nouveaux noyaux peuvent alors se former les uns à partir des autres :

- soit par la capture d'un neutron, il y a alors augmentation de la masse du noyau, l' isotope ainsi formé est plus exotique en neutrons :

- soit par émission d' un rayonnement $\beta^{-}$, l'élément ainsi formé gagne un proton, tout en gardant la même masse, il est donc plus stable.

La compétition entre ces deux processus dépend des taux de capture neutronique et des périodes radioactives des noyaux considérès.

Une expérience a élucidé le mystère de l'isotope du Calcium de masse 46 : ce dernier est en effet beaucoup moins abondant dans le système solaire que l'autre isotope du Calcium de masse 48 , bien qu'il soit plus proche du Calcium de masse 40 , le plus abondant dans la nature. La mesure des périodes et des taux d' émission des particules de radioactivité émis par des noyaux exotiques riches en neutrons a permis de déterminer les noyaux progéniteurs des 
isotopes de Calcium de masse 46 - 48, et ainsi de comprendre la raison de ces abondances à priori anormales.

Les astrophysiciens pour valider leurs théories ont besoin de davantage de données nucléaires expérimentales tels que les taux des réactions nucléaires, les masses, les périodes.... De nombreux programmes de recherches se développent pour mesurer ces données.

\section{DEMAIN, ACCÉLÉRER DES IONS RADIOACTIFS : LE PROJET SPIRAL.}

Les ions radioactifs produits par la fragmentation du projectile, collectés par le dispositif SISSI, puis triés par les différents spectromètres, forment d'ores et déjà des faisceaux radioactifs. S' ils ont déjà permis l'obtention de très nombreux résultats, ils ont cependant des limites :

- Une énergie élevée (celle du faisceau primaire) difficilement modulable.

- Des caractéristiques de faisceaux médiocres dues à la nécessité de traverser de la matière pour trier ces ions.

- Une intensité relativement faible due à l'utilisation, pour la production de ces ions, de cibles minces.

Devant les nombreux résultats obtenus sur les noyaux exotiques et la forte demande de faisceaux d'ions radioactifs, les ingénieurs et physiciens nucléaires ont conçu le projet SPIRAL (S ystème de Production d'Ions Radioactifs Accélérés en Ligne) prévu pour être opérationnel en 1998 et cofinancé par le C.E.A., le C.N.R.S., et la région Basse Normandie.

Deux paramètres sont essentiels pour augmenter la production d'ions radioactifs : d'une part accroître l'intensité du faisceau primaire GANIL, projet T.H.I. (Transport des Hautes Intensités prévu pour 1996), d'autre part augmenter l'épaisseur de la cible de production.

Le projet SPIRAL est schématisé sur la figure 6 . Le faisceau incident, dit primaire, du GANIL est envoyé sur une cible épaisse qui arrête le faisceau. Les noyaux radioactifs sont formés soit par la fragmentation des noyaux projectiles, soit par la fragmentation des noyaux cibles. Ces ions radioactifs sont extraits de la cible par la forte température (environ $2000^{\circ} \mathrm{C}$ ) induite par l'arrêt du faisceau primaire. Ils sont ensuite ionisés dans une source, triés par un spectromètre, puis accélérés par le cyclotron. 
A la sortie du cyclotron, les énergies seront comprises entre 3 et $20 \mathrm{MeV}$ par nucléon, dépendant essentiellement du nombre atomique de l'ion d'intérêt et du rapport de sa masse sur sa charge électrique. Les intensités attendues dépendront, elles, fortement du noyau, selon qu'il sera plus ou moins loin de la stabilité.

\section{LA PHYSIQUE NON NUCLÉAIRE AU GANIL.}

Outre l'aspect recherche fondamentale en physique nucléaire, le GANIL est aussi un outil très utilisé par les chercheurs qui étudient l'interaction particule-matière, la physique atomique et la matière condensée, comme en témoigne l'activité fertile du CIRIL (C entre Interdisciplinaire de $\mathbf{R}$ echerche avec des Ions Lourds) installé sur le site du GANIL. Les ions multi-chargés, constitués d'un très petit nombre d'électrons, sont étudiés d'une part pour leur structure atomique très particulière (recherche d'effets liès à la relativité et à l'électrodynamique quantique), d'autre part en ce qui concerne leur interaction importante, due à leurs fortes efficacité d'ionisation, avec les autres atomes (excitation, ionisation, échange de charge électrique, création de défauts dans les matériaux, canalisation des ions lourds selon un axe cristallographique...).

Le GANIL est aussi fortement engagé dans la Recherche et le Développement Industriel. Le Service des Applications Industrielles des Faisceaux (S.A.I.F.) étudie les conditions d'irradiation spécifiques aux impératifs industriels et les met en oeuvre; Il propose des prestations techniques basées sur son savoir-faire et gère les transferts de technologie.

La grande variété des faisceaux et de leurs caractéristiques (nature, énergie, dose, surface irradiée) permet déjà :

- La production de membranes à pores micrométriques.

- La simulation de l'environnement spatial pour améliorer la résistance des composants électroniques embarqués dans les satellites (Collaboration MATRA MARCONI SPACE et C.E.A./DAM /BIII/EL).

D'autres activités sont en cours de développement :

- L 'évaluation des dégâts causées par l' irradiation neutronique ou le vieillissement des aciers utilisés dans les réacteurs à eau pressurisée (Collaboration CIRIL-EDF).

- L'étude de faisabilité pour la réalisation d'écrans plats luminescents à micro pointes de grande dimension par lithographie ionique ( Collaboration C.E.A./DTA/LETI).

- L' implantation d'ions radioactifs (notamment le Béryllium de masse 7) pour des études de tribologie (Collaboration C.E.A./DTA/DAMRI). 
- L' amélioration des propriétés d'adhérence d'éléments à plusieurs couches.

\section{GANIL : ACCÉLÉRATEUR DE POINTE.}

En 12 ans de fonctionnement, le GANIL s'est acquis une solide renommée internationale par ses résultats tout à fait originaux dans un domaine où la compétition est vive. Le GSI (Gesellschaft für SchwerIonen) en Allemagne, Le National Superconducting Cyclotron laboratory aux États-Unis et le Riken au Japon sont ses principaux concurrents à travers le monde.

Depuis sa construction, il s'est remis sans cesse en question en construisant des dispositifs expérimentaux toujours plus performants: Le détecteur à particules multichargées INDRA, le détecteur à neutrons ORION, le détecteur à photons, pions et kaons TAPS, le filtre de Wien LISE3, le dispositif de collection SISSI.

Les faisceaux d'ions radioactifs suscitent un grand intérêt, comme en témoigne la dizaine de projets mondiaux, que ce soit en physique nucléaire, pour toutes les études relatives à l'influence de la dissymétrie entre le nombre de neutrons et de protons dans le noyau, que pour la physique non nucléaire.

Le GANIL a ainsi montré sa capacité à évoluer pour affronter de nouveaux défis scientifiques et garder sa place d'accélérateur de pointe. 


\section{Figures:}

Fig. 1 : L'accélérateur GANIL et ses aires expérimentales.

Fig. 2 : Le détecteur INDRA pour la recherche des noyaux chauds dans leurs états extrêmes.

Fig. 3 : Identification de noyaux exotiques :

- a) cette carte a pour coordonnées le rapport masse du noyau sur sa charge (axe horizontal) et le nombre atomique de chaque noyau (axe vertical); indiqués

- b) partie de la carte a) avec deux groupes d'isotopes d'étain

- c) et d) distributions en masse des régions sélectionnées sur la carte b) : pour chaque groupe sélectionné correspondent plusieurs isotopes d'étain dans différents états de charge dont le noyau doublement magique Étain de masse 100.

Fig. 4 : Comparaison des masses atomiques, mesurées, de différents isotopes d'oxygène à celles, calculées, à partir de plusieurs modèles théoriques.

Fig. 5 : Représentation schématique d'un noyau à halo. Certains noyaux légers très riches en neutrons présentent un halo : les neutrons excédentaires s'aventurent loin du "coeur" de nucléons, bien au delà des dimensions nucléaires usuelles.

Fig. 6 : Schéma de principe du projet SPIRAL (Systéme de Production d'Ions $\mathbf{R}$ adioactifs $\mathbf{A}$ ccélèrés en Ligne). 


\section{GANIL \\ Accélérateur et Aires Expérimentales}

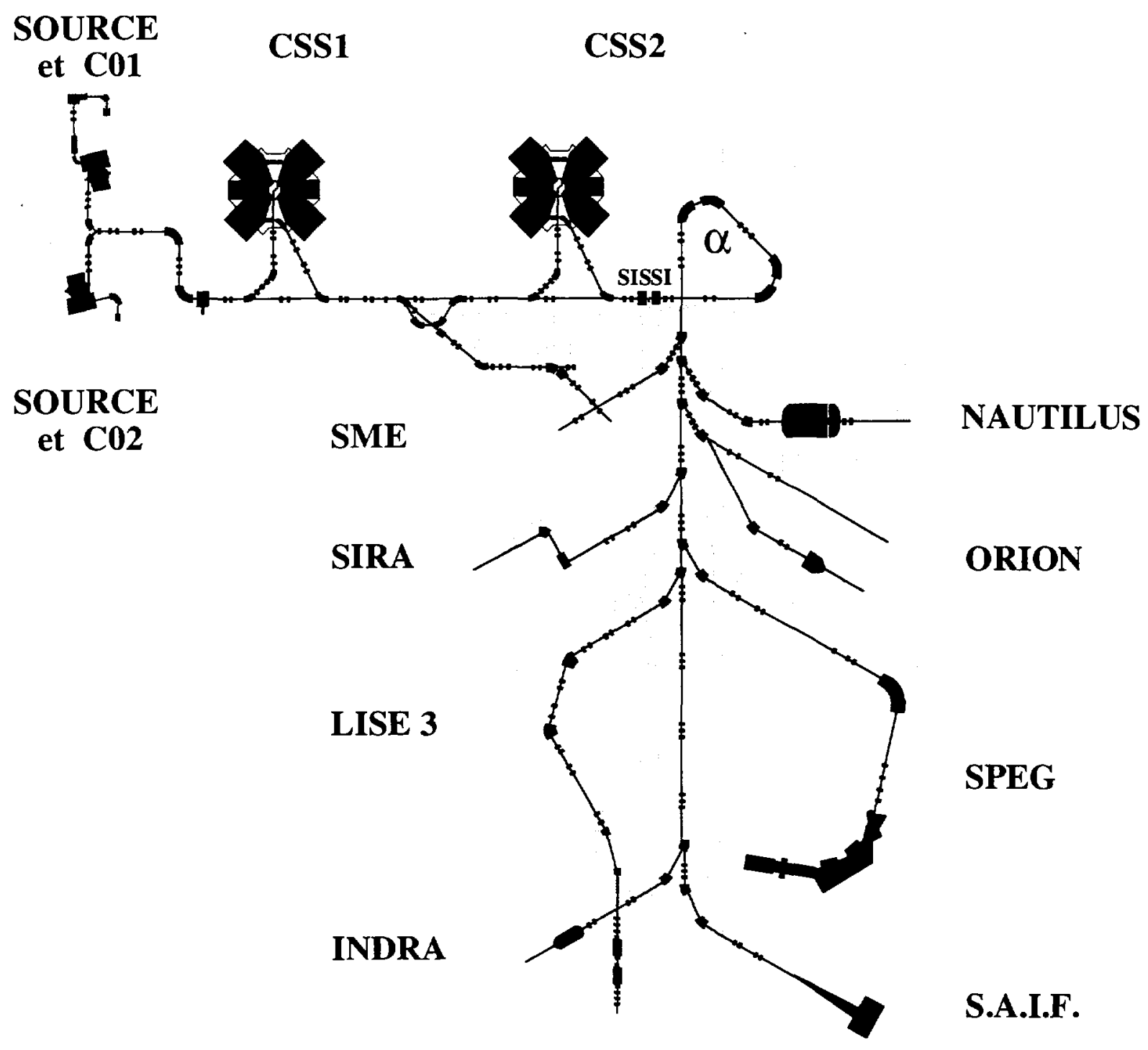




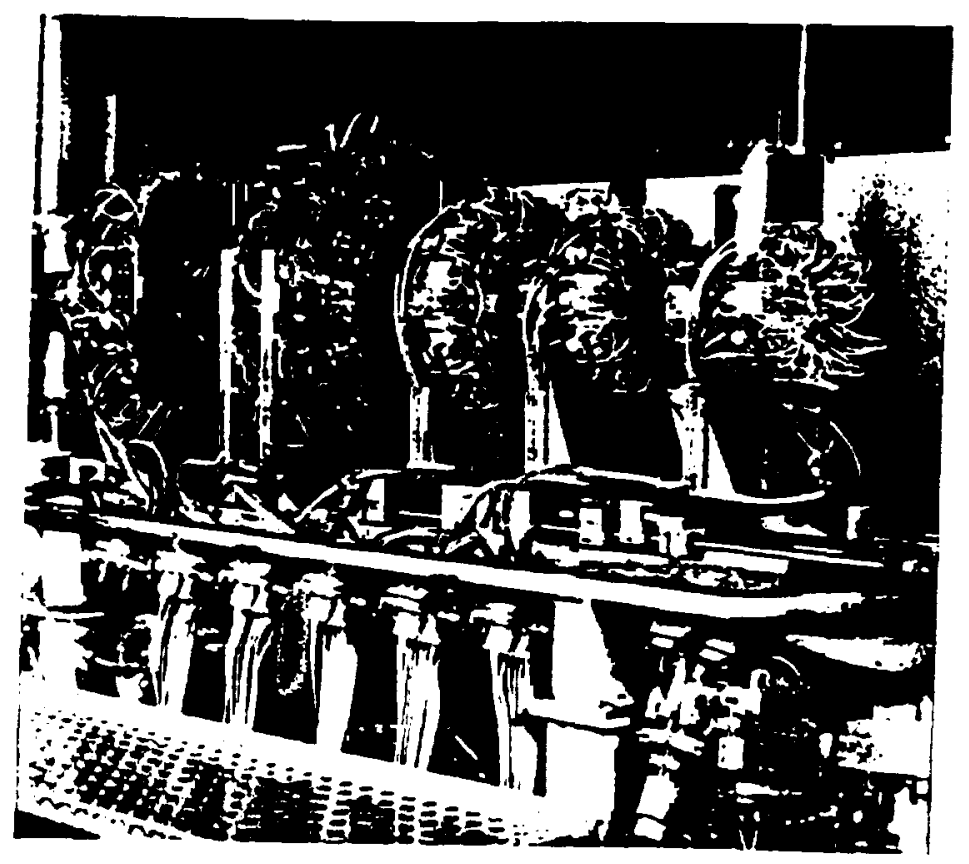



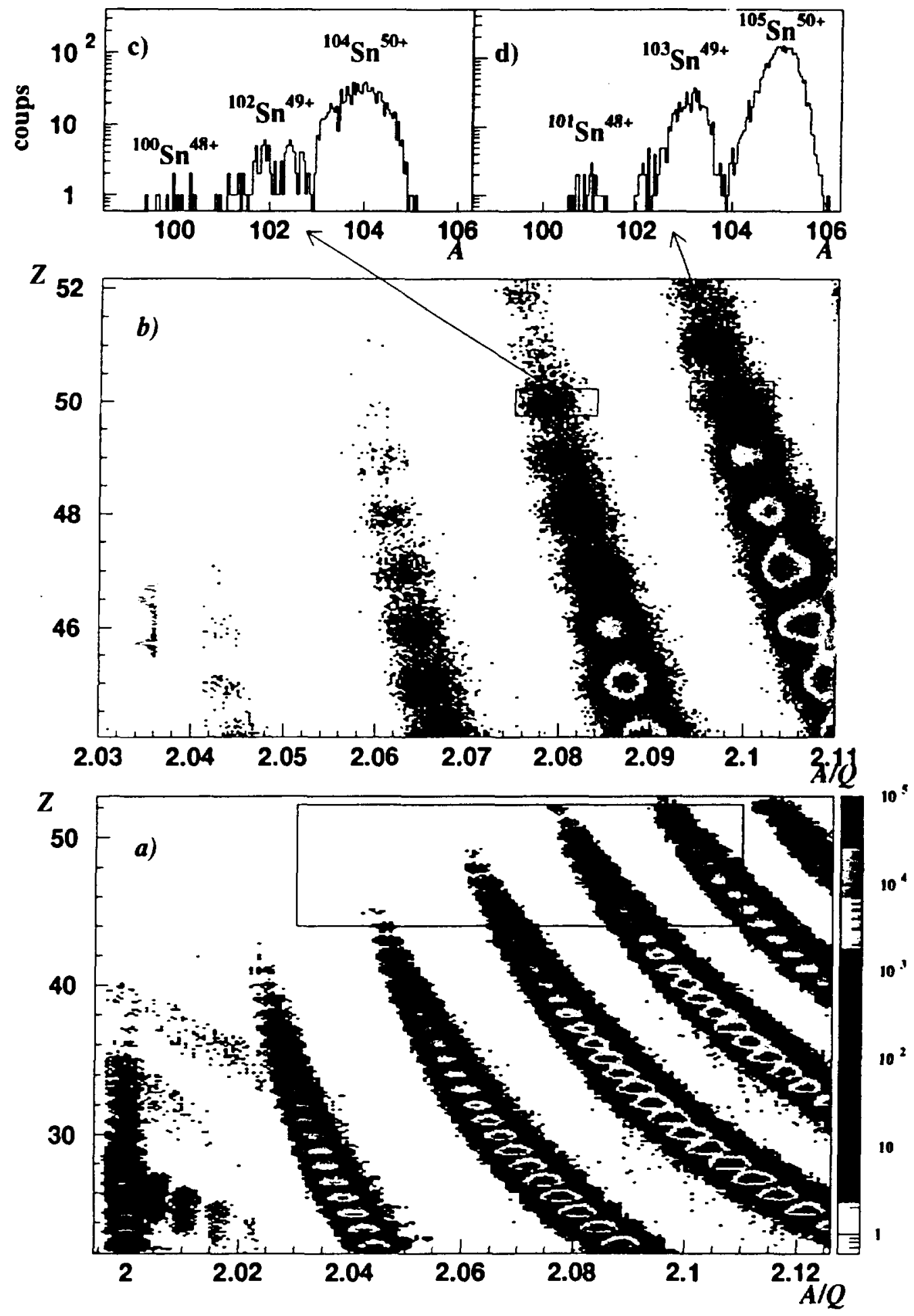


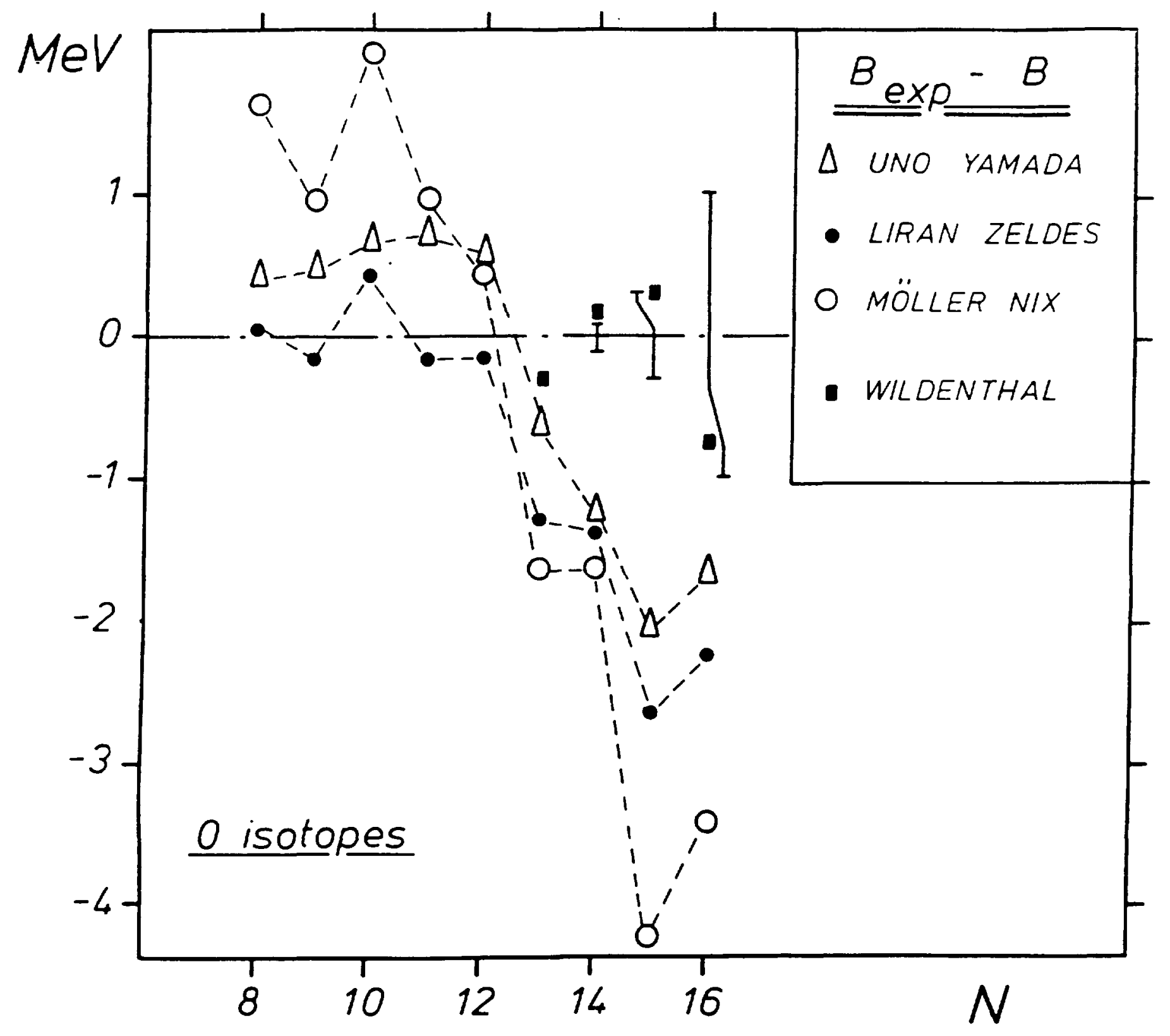





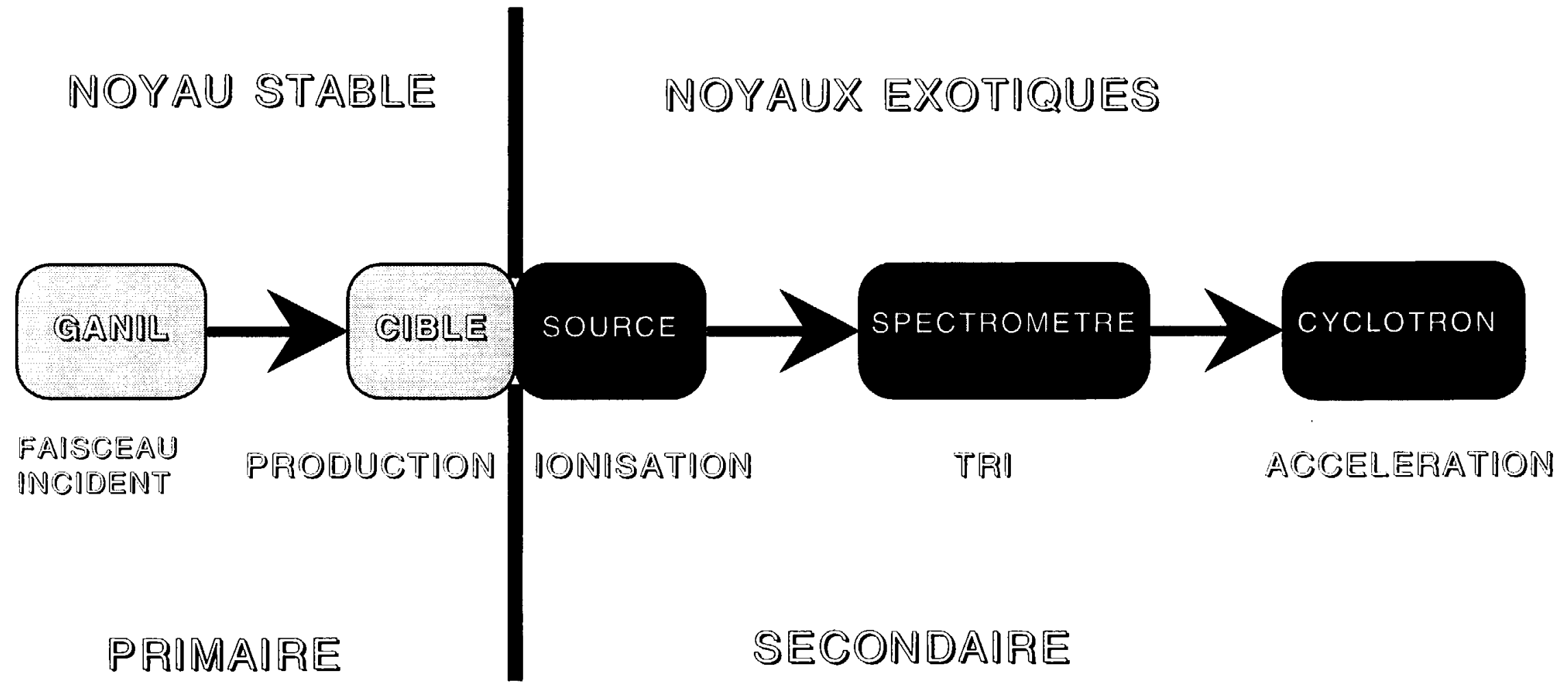

